\title{
PET/CT Respiratory Motion Correction With a Single Attenuation Map Using NAC Derived Deformation Fields
}

\author{
Alexander C. Whitehead, Student Member, IEEE, Ander Biguri, Nikos Efthimiou, Member IEEE, Kuan-Hao Su, \\ Scott D. Wollenweber, Senior Member IEEE, Charles W. Stearns, Fellow, IEEE, \\ Brian F. Hutton, Senior Member, IEEE, Jamie R. McClelland and Kris Thielemans, Senior Member, IEEE
}

\begin{abstract}
Respiratory motion correction is beneficial in positron emission tomography. Different strategies for handling attenuation correction in conjunction with motion correction exist. In clinical practice, usually a single attenuation map is available, derived from computed tomography in one respiratory state. This can introduce an unwanted bias (through misaligned anatomy) into the motion correction algorithm. This paper builds upon previous work which suggested that non-attenuation corrected data was suitable for motion estimation, through the use of motion models, if time-of-flight data are available. Here, the previous work is expanded upon by incorporating attenuation correction in an iterative process. Non-attenuation corrected volumes are reconstructed using ordered subset expectation maximisation and used as input for motion model estimation. A single attenuation map is then warped to the volumes, using the motion model, the volumes are attenuation corrected, after which another motion estimation and correction cycle is performed. For validation, 4Dimensional Extended Cardiac Torso simulations are used, for one bed position, with a field of view including the base of the lungs and the diaphragm. The output from the proposed method is evaluated against a non-motion corrected reconstruction of the same data visually, using a profile as well as standardised uptake value analysis. Results indicate that motion correction of inter-respiratory cycle motion is possible with this method, while accounting for attenuation deformation.
\end{abstract}

\section{INTRODUCTION}

$\mathbf{R}$ ESPIRATORY motion reduces image resolution in Positron Emission Tomography (PET) by introducing blurring and mis-alignment artefacts [1]. Unless gated Computed Tomography (CT) are available (which themselves increase dose to the patient), to avoid mis-registration due to attenuation

Alexander C. Whitehead, Ander Biguri, Brian F. Hutton and Kris Thielemans are with the Institute of Nuclear Medicine, University College London, UK (contact: alexander.whitehead.18@ucl.ac.uk).

Alexander C. Whitehead and Jamie R. McClelland are with the Centre for Medical Image Computing, University College London, UK.

Nikos Efthimiou is with the Department Radiology, Perelman School of Medicine, University of Pennsylvania, USA.

Kuan-Hao Su, Scott D. Wollenweber and Charles Stearns are with Molecular Imaging and Computed Tomography Engineering, GE Healthcare, USA.

This research is supported by GE Healthcare, the NIHR UCLH Biomedical Research Centre and the UCL EPSRC Centre for Doctoral Training in Intelligent, Integrated Imaging in Healthcare (i4health) grant (EP/L016478/1) The software used was partly produced by the Computational Collaborative Project in Synergistic PET-MR Reconstruction, CCP PET-MR, UK EPSRC grant (EP/M022587/1) and the CCP on Synergistic Biomedical Imaging, CCP SyneRBI, UK EPSRC grant (EP/T026693/1).

Jamie R. McClelland is supported by a Cancer Research UK Centres Network Accelerator Award grant (A21993) to the ART-NET consortium and a CRUK Multi-disciplinary grant (CRC 521) mismatches, most existing Motion Corrected (MC) methods rely on pair-wise registration of gated Non-Attenuation Corrected (NAC) PET volumes [2], [3]. This is a challenging problem due to the low contrast and high noise of these volumes. Other MC methods can incorporate, directly, both $\mathrm{MC}$ and Attenuation Map (Mu-Map) estimation into reconstruction, however, these can be computationally expensive [4].

In our previous work we investigated the possibility of using a Motion Model (MM) for respiratory MC where the MM was derived from NAC PET. One of the advantages of using a MM approach over pair-wise registering the data is that the $\mathrm{MM}$ approach is more robust to noise in the images. We found that NAC Time-of-Flight (TOF) PET was suitable to estimate the motion from gated PET data without inter-respiratory cycle variation [5]. This work extends the method towards attenuation correction with a single Mu-Map (from any position).

\section{METHODS}

\section{A. XCAT Volume Generation}

4-Dimensional Extended Cardiac Torso (XCAT) [6] was used to generate 240 volumes over a $120 \mathrm{~s}$ respiratory trace (with inter-respiratory cycle variation) derived from data captured using a Real Time Position Management (RPM). The max displacement of Anterior Posterior (AP) and Superior Inferior (SI) motion was set to $1.2 \mathrm{~cm}$ and $2.0 \mathrm{~cm}$ respectively. Activity concentrations were derived from a static Fluorodeoxyglucose (FDG) patient scan. The Field Of View (FOV) included the base of the lungs, diaphragm and the top of the liver with a $20 \mathrm{~mm}$ diameter spherical lesion placed into the centre of the right lung.

\section{B. PET Acquisition Simulation}

PET acquisitions were simulated (and reconstructed) using Software for Tomographic Image Reconstruction (STIR) [7][9] through the Synergistic Image Reconstruction Framework (SIRF) [10] to forward project the data using the geometry of a General Electric (GE) Discovery 710 with a TOF resolution of 375 ps. This TOF resolution is higher than that of the 710 , but is closer to the newer GE Signa PET/MR system. TOF mashing was used to reduce computation time resulting in 13 TOF time bins of size $376.5 \mathrm{ps}$. Attenuation was included using the relevant $\mathrm{Mu}-\mathrm{Maps}$ generated by XCAT. Scatter and randoms 
were not taken into account. Multiple noise realisations were generated to simulate an acquisition over $120 \mathrm{~s}$, emulating a standard single bed position acquisition. A respiratory Surrogate Signal (SS) was generated using Principal Component Analysis (PCA) [11]. This was used to gate the data into 10 respiratory bins using displacement gating. For the purpose of the MM fitting, SS values were ascertained for the post-gated data by taking an average of the SS values of the data in each bin.

\section{Non-Attenuation Corrected Image Reconstruction}

Data were reconstructed without Attenuation Corrected (AC) using OSEM with two full iterations and 24 subsets. [12]. Volumes were post-filtered using a Gaussian blur with a kernel size of $6.4 \mathrm{~mm}$ full width half maximum.

\section{Motion Model Estimation}

The MM method, in this work, makes use of 3-Dimensional (3D) B-spline Control Point Grids (CPGs) with the corresponding warping operation denoted as $\mathbf{W}\left(\alpha_{t}\right)$, with $\alpha_{t}$ a vector with motion parameters at time $t$ and the breathing surrogate signal s:

$$
\forall t \in\left[1, n_{t}\right], \quad \alpha_{n, t}:=R_{1, n} s_{1, t}+R_{2, n}
$$

where $\alpha_{n, t}$ is the motion parameter $n$ at time point $t$ and $R_{i, n}$ are the model parameters where $i=[1,2]$. A generalised framework unifying image registration and respiratory MMs were used to estimate MMs and Motion Compensated Images (MCIs) [13]. Sum of squared differences was used as the similarity measure and bending energy was used as a penalty. The CPG spacing and penalty weight were tuned using a grid search.

\section{E. Attenuation Map Warping}

A Mu-Map close to the mean respiratory position was selected from the Mu-Maps generated by XCAT. This MuMap was then registered (using Normalised Mutual Information (NMI)) to the mean position NAC MCI generated using the MM. The MM was then used to generate Deformation Vector Fields (DVFs) for the SS values of each bin, which were then used to warp the Mu-Map from the mean respiratory position to each bin.

\section{F. Motion Corrected Image Reconstruction with AC}

Data were re-reconstructed, with AC, using the Mu-Maps from Section II-E. The same reconstruction parameters as in Section II-F were used. These data were then either motion corrected using the original NAC MM or a new MM was fit on the AC volumes as in Section II-D.

\section{G. Evaluation}

To evaluate the validity of the MM results, the Centre of Mass (COM) of the lesion, over time, was tracked for both $\mathrm{NAC}$ and $\mathrm{AC}$ reconstructions. This was achieved by warping a volume only including the lesion in the reference position, and then computing its COM.

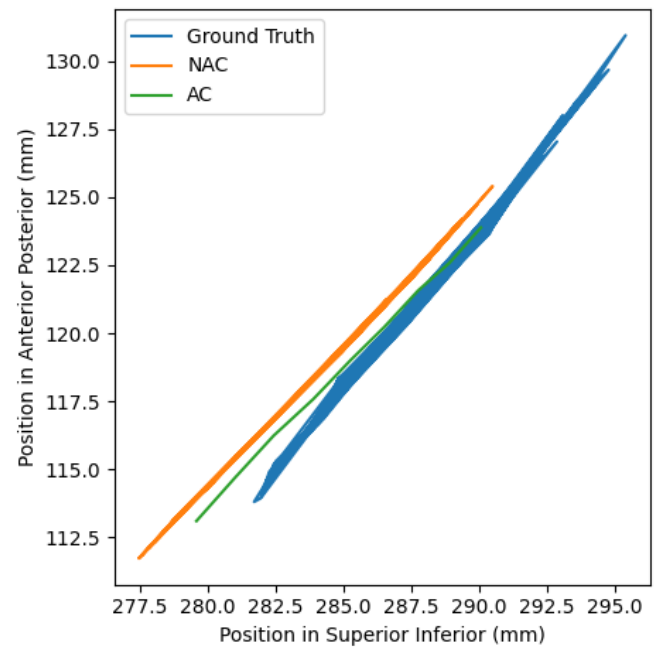

Fig. 1. The path of the COM of the lesion. Horizontal (respectively vertical) axis corresponds to motion in the SI (respectively AP). Different curves denote COM displacement for ground truth data, the estimated data from the NAC based MM and the estimated data from the AC based MM.

TABLE I

COMPARISON OF SUV MAX, SUV MEdAN AND SUV PEAK BETWEEN; UNGATED (CINE-CT), UNGATED (STATIC CT), NAC MM, AC $\mathrm{MM}$.

\begin{tabular}{||c|ccc||}
\hline SUV & Max & Median & Peak \\
\hline Ungated (CINE-CT) & 4.63 & 2.73 & 3.39 \\
Ungated (static CT) & 4.66 & 3.05 & 3.54 \\
\hline NAC MM & 5.56 & 3.18 & 4.07 \\
AC MM & 5.43 & 3.18 & 4.00 \\
\hline
\end{tabular}

In addition to the reconstructions performed in Section II-F data were also reconstructed after simply summing all gates together, using either a sum of all Mu-Maps (to emulate an average CINE-CT) or one Mu-Map, positioned close to the mean respiratory position. This process matches current clinical practice.

Comparisons used included: A profile over the lesion and Standard Uptake Value (SUV) $\max , \mathrm{SUV}_{\text {median }}$ and $\mathrm{SUV}_{\text {peak }}$. $\mathrm{SUV}_{\text {peak }}$ here was defined following European Association of Nuclear Medicine (EANM) guidelines [14].

\section{RESULTS}

$\mathrm{COM}$ results can be seen in Fig. 1, the COM of both the NAC and AC matches closely the ground truth COM.

The ungated and the MM data can be seen in Fig. 2. When compared visually structures can be seen, less blurred, in the MM data that cannot be seen in the ungated data, for instance, structures at the boundary between the diaphragm and the lung. The different levels of blurring in the ungated (CINECT) and static CT could be attributed to the constraint put on the reconstruction by having a sharp Mu-Map in one respiratory position in the static CT case. Additionally the lesion itself can be seen to be more homogeneous, this can be observed in the profile across the lesion in Fig. 3. SUV results can be seen in TABLE I and consistently show that SUVs are greater for the MM over the ungated method. 


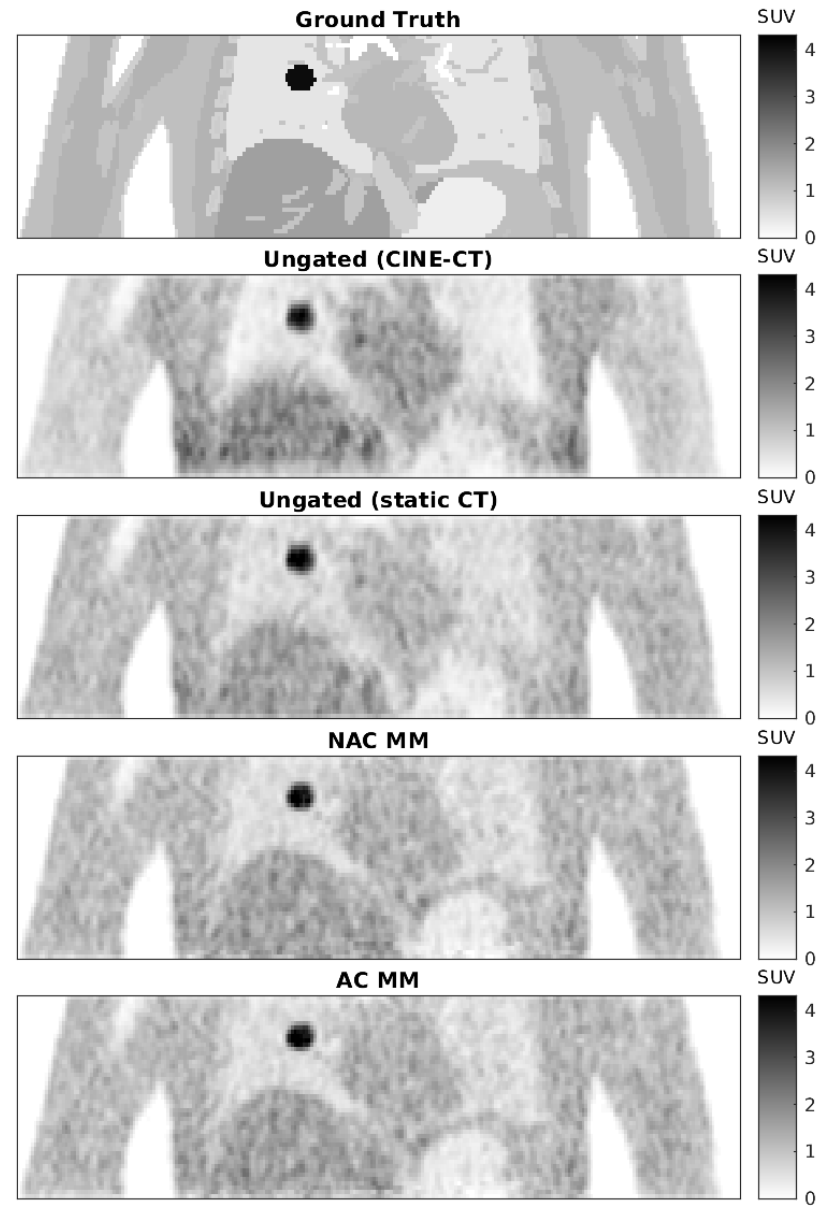

Fig. 2. Ground truth and reconstructions using; ungated (CINE-CT), ungated (static CT), NAC MM, AC MM. Colour map ranges are consistent for all images.

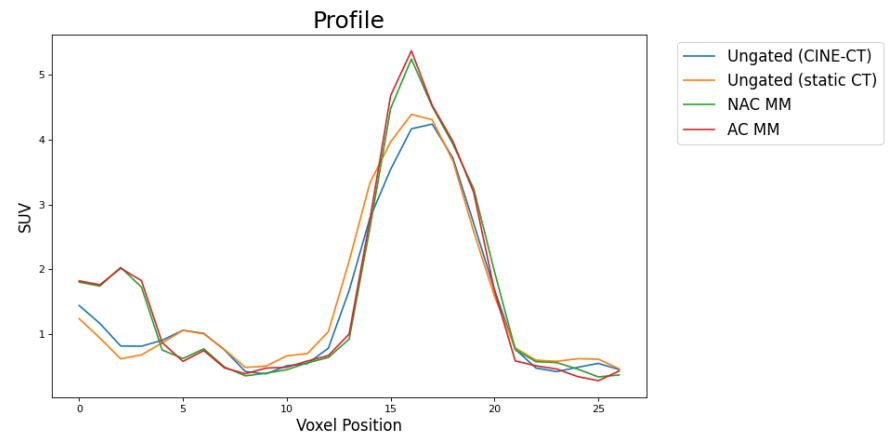

Fig. 3. A profile across the lesion for; ungated (CINE-CT), ungated (static CT), NAC MM, AC MM.

\section{DISCUSSION AND CONCLUSIONS}

Results from both a visual analysis, a comparison of profiles and SUVs show that the MM provides volumes more free from blurring and less susceptible to artefacts when compared to the ungated data. Results also indicate that the NAC MM provides similar volumes while not requiring the additional computation of the AC MM.

In the future, we will incorporate the estimated DVFs into the reconstruction. We will also compare more complex methods of combining motion estimation and image reconstruction based on [4]. We also hope to find better results from improvements to TOF resolution [15], [16].

\section{REFERENCES}

[1] S. A. Nehmeh et al., "Respiratory Motion in Positron Emission Tomography/Computed Tomography: A Review," Seminars in Nuclear Medicine, vol. 38, no. 3, pp. 167-176, May 2008.

[2] W. Bai et al., "Motion correction and attenuation correction for respiratory gated PET images," IEEE Transactions on Medical Imaging, vol. 30, no. 2, pp. 351365, Feb. 2004.

[3] F. P. Oliveira et al., "Medical image registration: a review," Computer Methods in Biomechanics and Biomedical Engineering, vol. 17, no. 2, pp. 73-93, Jan. 2014.

[4] A. Bousse et al., "Maximum-likelihood joint image reconstruction and motion estimation with misaligned attenuation in TOF-PET/CT," Physics in Medicine and Biology, vol. 61, no. 3, pp. L11-L19, Feb. 2016.

[5] A. C. Whitehead et al., "Impact of Time-of-Flight on Respiratory MotionModelling using Non-Attenuation-Corrected PET," in IEEE Nuclear Science Symposium and Medical Imaging Conference (NSS/MIC), Manchester, 2019.

[6] W. P. Segars et al., "4D XCAT phantom for multimodality imaging research," Medical Physics, vol. 37, no. 9, pp. 4902-4915, Aug. 2010.

[7] K. Thielemans et al., "STIR: software for tomographic image reconstruction release 2," Physics in Medicine and Biology, vol. 57, no. 4, pp. 867-883, Feb. 2012.

[8] N. Efthimiou et al., "Implementation and validation of time-of-flight PET image reconstruction module for listmode and sinogram projection data in the STIR library," Physics in Medicine and Biology, vol. 64, no. 3, p. 035004 , Jan. 2019.

[9] P. Wadhwa et al., "PET image reconstruction using physical and mathematical modelling for time of flight PET-MR scanners in the STIR library," Methods, 2020.

[10] E. Ovtchinnikov et al., "SIRF: Synergistic Image Reconstruction Framework," in 2017 IEEE Nuclear Science Symposium and Medical Imaging Conference (NSS/MIC), IEEE, Oct. 2017, pp. 1-3.

[11] K. Thielemans et al., "Device-less gating for PET/CT using PCA," IEEE Nuclear Science Symposium Conference Record, pp. 3904-3910, 2011.

[12] H. M. Hudson et al., "Accelerated Image Reconstruction Using Ordered Subsets of Projection Data," IEEE Transactions on Medical Imaging, vol. 13, no. 4, pp. 601-609, 1994.

[13] J. R. McClelland et al., "A generalized framework unifying image registration and respiratory motion models and incorporating image reconstruction, for partial image data or full images," Physics in Medicine and Biology, vol. 62, no. 11, pp. 4273-4292, Jun. 2017.

[14] R. Boellaard et al., FDG PET/CT: EANM procedure guidelines for tumour imaging: version 2.0, 2015.

[15] N. Efthimiou et al., "Use of non-Gaussian time-of-flight kernels for image reconstruction of Monte Carlo simulated data of ultra-fast PET scanners," EJNMMI Physics, vol. 7, no. 1, p. 42, Dec. 2020.

[16] N. Efthimiou et al., "TOF-PET image reconstruction with multiple timing kernels applied on Cherenkov radiation in BGO,” Tech. Rep., Oct. 2020, pp. 1-9. 